\title{
Generating multimedia presentations that summarize the behavior of dynamic systems using a model-based approach
}

\author{
Martin Molina*, Victor Flores \\ Department of Artificial Intelligence, Technical University of Madrid, Campus de Montegancedo S/N, 28660 Boadilla del Monte, Madrid, Spain
}

\section{A R T I C L E I N F O}

\section{Keywords:}

Behavior summarization

Presentation model

Intelligent user interface

Data abstraction

Multimedia presentation

Text-graphic coordination

Data-to-text system

\begin{abstract}
A B S T R A C T
This article describes a knowledge-based method for generating multimedia descriptions that summarize the behavior of dynamic systems. We designed this method for users who monitor the behavior of a dynamic system with the help of sensor networks and make decisions according to prefixed management goals. Our method generates presentations using different modes such as text in natural language, 2D graphics and 3D animations. The method uses a qualitative representation of the dynamic system based on hierarchies of components and causal influences. The method includes an abstraction generator that uses the system representation to find and aggregate relevant data at an appropriate level of abstraction. In addition, the method includes a hierarchical planner to generate a presentation using a model with discourse patterns. Our method provides an efficient and flexible solution to generate concise and adapted multimedia presentations that summarize thousands of time series. It is general to be adapted to different dynamic systems with acceptable knowledge acquisition effort by reusing and adapting intuitive representations. We validated our method and evaluated its practical utility by developing several models for an application that worked in continuous real time operation for more than 1 year, summarizing sensor data of a national hydrologic information system in Spain.
\end{abstract}

(c) 2011 Elsevier Ltd. All rights reserved.

\section{Introduction}

The management of dynamic systems by means of sensor networks is a type of task that usually involves teams of operators and decision makers. In general, the human teams are interested not only in the interpretations of the values recorded by sensors, but also in the description of possible phenomena and the evolution of these in the time. An example of a dynamic system in the domain of hydrology is a set of geographically distributed river basins with river channels and reservoirs. Here, human operators monitor the state of the rivers and make decisions in the presence of problems such as floods, water contamination, or unexpected water needs (for agriculture, hydro-electrical energy or water consumption by end-users). Other examples of this type of dynamic system are: road traffic networks, public transport systems (by bus, railway, subway, etc.), electrical energy distribution systems, physical phenomena related to natural disasters (for example, seis-

\footnotetext{
* Corresponding author. Address: Departamento de Inteligencia Artificial, Facultad de Informática, Universidad Politécnica de Madrid, Campus de Montegancedo S/N, 28660 Boadilla del Monte, Madrid, Spain. Tel.: +34 913367390; fax: +34 913524819.

E-mail addresses: martin.molina@upm.es (M. Molina),victor.flores@fi.upm.es (V. Flores).
}

mic or volcano activity), historical records of vehicle behavior (e.g., a military aircraft) for maintenance purposes, etc.

Tools that automatically generate descriptions and explanations about the system's behavior can help end-users understand and analyze the meaning of data. This is especially important when the dimension and complexity of the system is high with large amounts of quantitative data from sensors that record different types of magnitudes periodically. To generate such type of descriptions, it is necessary to select and abstract automatically relevant sensor data and generate adequate interpretations. In addition, it is necessary to dynamically construct discourses at an adequate level of abstraction that is useful for end-users according to their information needs. The effective simulation of these reasoning processes requires representing specialized domain knowledge about the dynamic system in a practical way, i.e., able to be interpreted efficiently by inference methods and able to be acquired with acceptable effort.

A number of research projects have paid attention (directly or indirectly) to this type of problem. For example, SumTime generates text descriptions that summarize temporal series about weather forecasts (Reiter, Sripada, Hunter, Yu, \& Davy, 2005), SumGen generates text summaries of a set of events from a battle simulator (Maybury, 1995) and the method of Gautier and Gruber generates text explanations about the behavior of a space shuttle's reaction control system (Gruber \& Gautier, 1993). However, a limitation of these solutions is that they generate only text descriptions. In 
complex dynamic systems, it is important to use also coordinated illustrations that complement text for a better understanding of behavior data (e.g., map locations, graphics for temporal series, etc.). Another problem of existing proposals (as it happens, for example, in SumGen and SumTime) is that they use specific domain knowledge bases for data abstraction and text generation that are difficult to be reused for other dynamic systems.

In this article, we describe an innovative knowledge-based method to generate descriptions that summarize datasets corresponding to the observed behavior of dynamic systems. ${ }^{1}$ Our method is able to generate multimedia presentations about behavior (with graphics, images, animations) and, also, it is flexible to be adapted to different dynamic systems. Our method uses a general and reusable representation for the dynamic system especially designed to support strategies for summarizing data from thousands of sensors. This representation is based on approximate knowledge that can be obtained with acceptable knowledge acquisition effort. We validated our method and showed its practical utility by developing several models in the domain of hydrology that work in continuous operation using real-time sensor data from a national hydrologic information system in Spain.

In the following, we describe the method for generating descriptions that summarize behavior datasets. First, we present the summarization goals describing the general characteristics of the dynamic system. Next, we present the knowledge-based architecture and, then, we describe in detail the representation for dynamic systems and other representations and inferences for abstraction and presentation planning. We also describe how we validated our method and evaluated its practical utility. At the end of the article, we make a comparative discussion with similar approaches.

\section{Summarization goals}

Our method generates descriptions that summarize the behavior of a certain type of dynamic system. The general characteristics of such dynamic system are the following:

- Observable. The behavior of the system is observed with the help of sensors that periodically measure the value of a number of quantitative properties. There are different types of sensors that observe different types of quantities. There may be a large number of quantities whose value change in time.

- Complex. The dynamic system has different types of components and relations among components. The system can be described at different levels of aggregation with parts that, in turn, can be decomposed in smaller parts.

- Supervised. There are human teams that supervise the system's behavior and make decisions accordingly. For example, there are human operators in charge of control that watch the system and, if necessary, change its state with the help of actuators. In addition, there are other types of users that can be affected by the state of the system. They are interested in the system's behavior to make decisions about themselves and other related systems.

- Prefixed goals. The system is controlled according to prefixed management goals that try to keep certain indicators about the state of the system close to acceptable values.

- Abstract view for decision. There is an important distance between (1) the language (and level of detail) in which sensors measure the quantities of the system and (2) the language (and level of abstraction) in which users make decisions.

\footnotetext{
1 Some partial preliminary results related to the research work presented in this article were previously reported in (Molina \& Flores, 2006a, 2006b, 2008).
}

The input to our method is a set of measures recorded by sensors. Each measure corresponds to an observed quantity of the dynamic system (for example the temperature of a particular component). For each quantity there is a time series with the last $t$ values (for example, $t=24$ ). We assume that each measure is recorded periodically, every $\Delta t$ (for example, $\Delta t=15 \mathrm{~min}$ ). We also assume that for a particular dynamic system there is a large number $N$ of sensors (for example, $N>1000$ ).

The output of the method is a description that summarizes and explains the behavior of the dynamic system. The description includes text summaries together with other visualization modes (2D graphics, maps, animations, 3D images, etc.) that could be presented using different devices (a computer desktop, a mobile phone, a fax, etc.). The method must include a solution to coordinate adequately the text and the appropriate illustrations that help understand quickly the information. This is especially important for dynamic systems with many components that need to be identified not only by their name but also by spatial location (for example, using a descriptive sketch of an installation, or using a geographic map). It is also useful to show the temporal change of different components with comparative graphics for temporal series, or using animations (2D or 3D animations) that help to understand the evolution of certain phenomena. To generate such type of descriptions, we identified the following challenges:

- Relevant information. The generated description must include the relevant information. Filtering thousands of quantitative measures to select the relevant information usually requires specific knowledge about both the historical behavior of the dynamic system and what is potentially useful for the end-user.

- Meaningful descriptions. The summary must explain the meaning of the measures in a qualitative language close to the enduser. This requires knowledge to interpret the quantitative data using appropriate meaningful qualitative linguistic labels.

- Variable level of abstraction. The summary must present condensed information as concise as possible. The level of abstraction to describe the behavior is not prefixed but it is dynamically chosen according to the input data and length restrictions on the output description. Condensing information requires knowledge such as how to generalize similar facts, how to aggregate facts corresponding to components that are part of more complex components and how to condense different facts corresponding to the same phenomenon.

- Variable discourse. The discourse structure of the descriptions is not rigid, but is dynamically constructed according to characteristics of the input data and the desired length of the presentations. The specific natural language sentences and their order in paragraphs is dynamically chosen together with the adequate illustrations that complement the descriptions.

- Convincing descriptions. The presentation needs to include evidence that helps users to trust the generated descriptions. This is important for users who have to assume the responsibility for decision-making based on these descriptions. The method needs to know strategies to select and present appropriate evidences to construct convincing descriptions.

\section{The knowledge-based architecture}

Our method follows a knowledge-based approach with a set of general inference steps that use domain specific knowledge. The method performs two main tasks: (1) abstraction, to abstract the relevant information (i.e., what to inform) and (2) planning, to generate a presentation plan according to the type of end-user and the communication media (how to present the information). Fig. 1 shows the components of our method with the two main tasks, abstraction and planning, together with inputs/outputs and the domain 


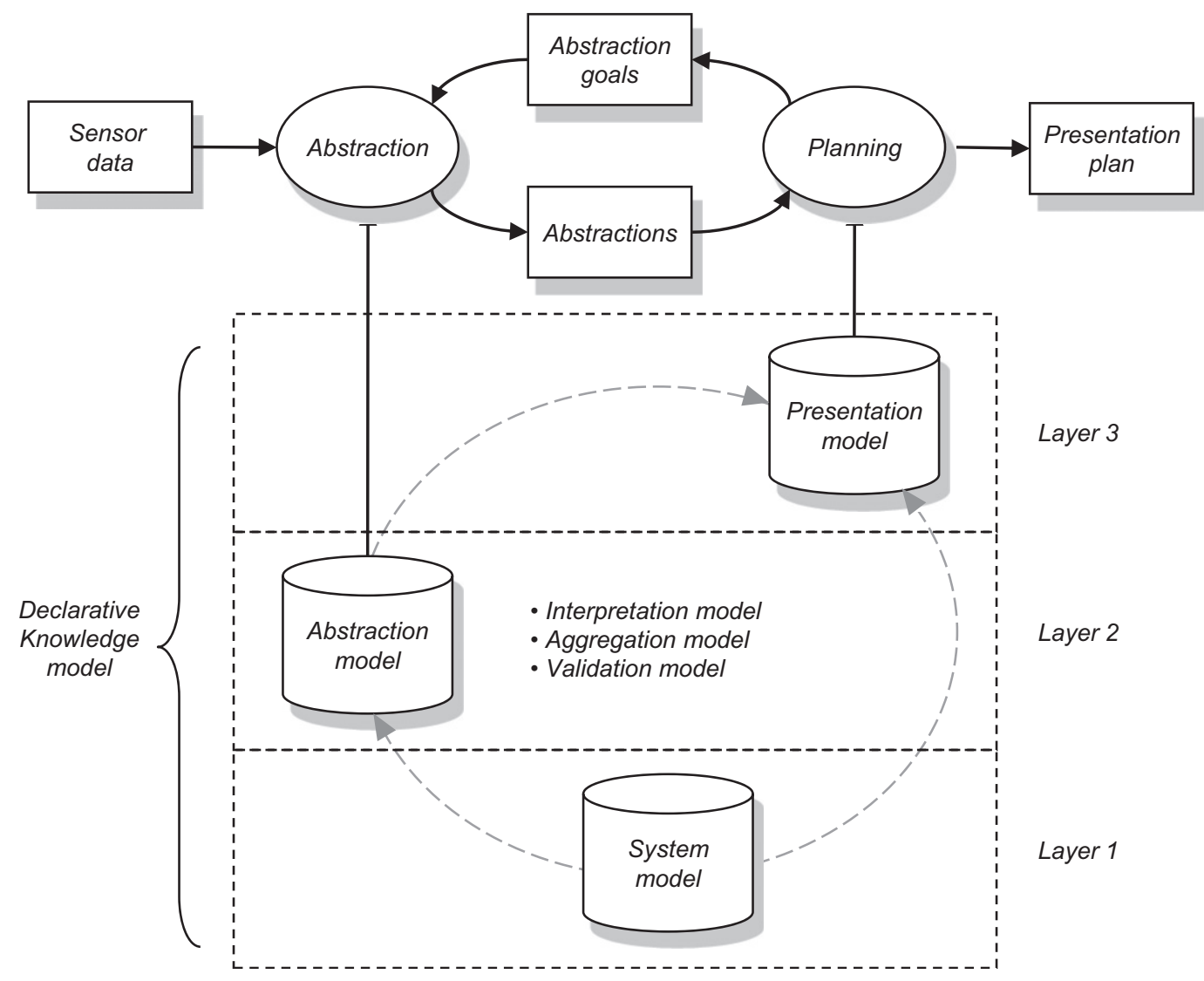

Fig. 1. Main components of our method. In the figure ellipses represent tasks, rectangles represent input/output data and cylinders represent knowledge bases.

knowledge. As the figure shows, the declarative knowledge representation is organized in meta-levels with three related layers:

(1) System model. The system model (layer 1) represents knowledge about the structure and behavior of the dynamic system.

(2) Abstraction model. The abstraction model (layer 2) represents knowledge about how to generate abstractions. It uses knowledge representations from layer 1.

(3) Presentation model. The presentation model (layer 3) represents knowledge about presentation strategies. It uses knowledge representations from layers 1 and 2.

Briefly, the method works as follows. We generate the presentation plan in a loop involving the two main tasks (planning and abstraction). The planning task performs a hierarchical search over candidate partial presentations using the presentation model. At each iteration, the planning task generates abstraction goals and the abstraction task uses the abstraction model to return the abstractions corresponding to these goals. With this information, the planning task progressively refines the partial presentations until the final presentation plan is constructed. In the following, we describe the three domain models and inference processes in more detail.

\section{The system model}

The system model is a representation of the behavior and the structure of the dynamic system. Our method is designed to simulate summarization tasks performed by professional human operators with partial and approximated knowledge about the dynamic system. Thus, the system model is formulated with a qualitative approach instead of precise mathematical representations with quantitative parameters. Our representation for the system model shares certain elements of existing common representations and ontologies used in qualitative physics (Bobrow et al., 1996; Borst, Akkermans, Pos, \& Top, 1995; Gruber \& Olsen, 1994; Iwasaki \& Low, 1993).

Compared to existing qualitative approaches for system modeling, our representation is not for simulation but, instead, for summarization, a task that in general requires less details and, therefore, it uses knowledge that can be obtained with lower knowledge acquisition effort. Our representation follows an intuitive component-based approach to perform abstraction processes such as aggregation, generalization and qualitative interpretation. This is done by using hierarchies of components (both part-of and is- $a$ hierarchies), qualitative states and causal influences.

We formalize the system model in many-sorted first-order logic (Meinke \& Tucker, 1993), an extension of first-order logic where sorts are specified for each constant, variable, function symbol and each argument position of each predicate and function symbol. Our representation for the system model uses the following basic sorts: component represents a physical object of the system (for example, a reservoir or a river), quantity is a quantitative property of a component (e.g., the temperature or the pressure), and sensor is a device used to measure observable quantities of components. More specific components are related to more general components (with the is- $a$ relation) by defining subsorts with the notation sort $s$ : $t$ (where $s$ is subsort of $t$ ). For example, sort reservoir: component defines the subsort reservoir of the sort component.

To represent qualitative properties we use the following sorts: state represents the qualitative state of a component at the present moment (for example, the current state of a reservoir is empty), recent_state represents the state of a component in a recent time interval (e.g., the last $24 \mathrm{~h}$ ), trend represents the trend of a state (for example, with the set of values \{increasing, steady, decreasing\}), 
and quantification is a sort that quantifies a state in a population of components (for example, with the set of values \{all, many, few, one, zero\}). The recent state is a practical solution to summarize a recent behavior in a qualitative value and it is usually described in a more abstract level than the current state.

In order to characterize adequately generalization levels, our representation also includes the scope of affirmations. For example, we want to represent that the affirmation the temperature in Spain is $32^{\circ}$ corresponds to a certain spatio-temporal scope such as the following: maximum value of the last $24 \mathrm{~h}$ and maximum value of the temperature in the main cities of Spain. For this purpose, we use the concept of relative scope to a specific domain. We use two sorts: $t_{-}$scope, which defines a temporal scope, and c_scope, which defines the scope in a set of subcomponents that are part of a given component. Table 1 shows possible values for these two sorts.

Table 2 shows a list of predicates to represent knowledge about the dynamic system. To represent structural relations, we use the predicate part_of( $x$ : component, $y$ : component) for the part-of relation and the predicate observe( $x$ : sensor, $y$ : quantity, $z$ : component) to represent that a quantity of a component is observed (measured) by a sensor. According to the part-of relation, component $c 1$ is complex when there exists another component $c 2$ and it is defined the relation part_of $(c 2, c 1)$. In contrast, a component is single when this property is no satisfied. The predicate state_category $(x$ : component, $y$ : state, $z$ : state) defines general states that correspond to categories of more specific states.

The model includes also an approximate view of the system behavior represented with causal relations between quantitative properties. This intuitive relation is useful to summarizing data

Table 1

Examples of possible values for the sorts $t_{-}$scope y $c_{-}$scope.

\begin{tabular}{|c|c|c|}
\hline Sort & Value & Description \\
\hline t_scope & $\begin{array}{l}\text { series }(n) \\
\text { current } \\
\text { maximum }(n) \\
\text { minimum }(n) \\
\text { average }(n) \\
\text { sum }(n) \\
\text { maximum } \\
\text { minimum } \\
\text { average } \\
\text { forecast }(n)\end{array}$ & $\begin{array}{l}\text { A temporal series with the last } n \text { values } \\
\text { The value at present moment } \\
\text { The maximum of the last } n \text { values } \\
\text { The minimum of the last } n \text { values } \\
\text { The average of the last } n \text { values } \\
\text { The sum of the last } n \text { values } \\
\text { The historical maximum value } \\
\text { The historical minimum value } \\
\text { The historical average value } \\
\text { A temporal series with a prediction of the future } n \\
\text { values }\end{array}$ \\
\hline c_scope & $\begin{array}{l}\text { maximum } \\
\text { minimum } \\
\text { average } \\
\text { sum }\end{array}$ & $\begin{array}{l}\text { The maximum value in the set of the components } \\
\text { The minimum value in the set of the components } \\
\text { The average value in the set of the components } \\
\text { The sum of values in the set of the components }\end{array}$ \\
\hline
\end{tabular}

Table 2

Predicates to represent knowledge about the dynamic system.

\begin{tabular}{ll}
\hline Predicate & Description \\
\hline $\begin{array}{l}\text { part_of }(x, y) \\
\text { observe }(x, y, z) \\
\text { cause }(x, y, z, u, t)\end{array}$ & $\begin{array}{l}\text { Component } x \text { is part of component } y \\
\text { Quantity } y \text { of component } z \text { is observed by sensor } x \\
\text { Quantity } x \text { of component } y \text { is cause of quantity } z \text { of } \\
\text { component } u \text { with the delay } t\end{array}$ \\
measurement $(x, y, z)$ & $\begin{array}{l}\text { Quantity } x \text { measured by sensor } y \text { has the value } z \text { (a } \\
\text { temporal series) } \\
\text { Quantity } x \text { of a single component } y \text { with t_scope } t \text { has } \\
\text { value }(x, y, t, v)\end{array}$ \\
the value $v$ & $\begin{array}{l}\text { Quantity } x \text { of a complex component } y \text { with t_scope } t \\
\text { and c_scope } z \text { has the value } v\end{array}$ \\
state $(x, y)$ & The state of component $x$ is $y$ \\
recent_state $(x, y)$ & The recent state of component $x$ is $y$ \\
trend $(x, y)$ & The trend of the state of component $x$ is $y$ \\
quantification $(x, y)$ & The quantification of the state of component $x$ is $y$ \\
state_category $(x, y, z)$ & The state $z$ is a category of the state $y$ of component $x$ \\
\hline
\end{tabular}

corresponding to the same phenomenon and generating explanations that help to understand data. The predicate cause ( $x 1$ : quantity, $y 1$ : component, $x 2$ : quantity, $y 2$ : component, $t$ : number) represents a direct causal influence between two quantities. The relation includes a temporal delay between the cause and effect.

To represent the value of a particular quantity we use the predicate value( $x$ : quantity, $y$ : component, $t: t \_s c o p e, v$ : value) for the case of a single component. This predicate defines the value for the quantity of a component with a particular temporal scope. For example, value(temperature, tank-T3, current, 120) represents that the temperature of tank-T3 is 120 at the present moment and value(volume, reservoir-R8, minimum $(24), 18$ ) represents that the minimum volume of reservoir-R8 in the last $24 \mathrm{~h}$ is 18 . The predicate for the case of complex components is value $\left(x\right.$ : quantity, $y$ : component, $t$ : $t_{-}$scope, $z$ : c_scope, $v$ : value). It includes an additional argument for c_scope. For example, value(rain, Spain, current, maximum, 27) represents that, at the present moment, the maximum rain in the set of points where rain is measured that are part of Spain is 27.

To interpret the current state of a component we use the predicates state( $x$ : component, $y$ : state), trend( $x$ : component, $y$ : state) and quantification( $x$ : component, $y$ : quantification). For example the tuple 〈state(Spain, heavy-rain), trend(Spain, decreasing), quantification(Spain, few) $\rangle$ represents that there is a decreasing heavy rain in a few points of Spain. The predicate recent_state( $x$ : component, $y$ : recent_state) represents the qualitative recent state of a component corresponding to a recent prefixed time interval.

\section{The abstraction model}

The abstraction model includes three submodels: the interpretation model, the relevance model and the validation model. In the following, we describe these models in detail. Then, we describe how we generate abstractions by using such models.

\subsection{The interpretation model}

The interpretation model represents knowledge that helps to determine the qualitative state of components. For the case of single components, their state is determined by conditions about quantities. The state of single components is similar to the qualitative values used in qualitative reasoning. For instance, the state of a reservoir may have the set of values \{empty, medium, full\}. We assume a total order in qualitative values. We obtain the state value of a single component by interpreting the numerical values of related quantities of the component, considering the limit points that define a quantitative space of a quantity (Forbus, 1984). For this, we use qualitative interpretation rules. The antecedent of such rules includes conditions about limit points in quantity values. For example, let consider the following rule:

sort reservoir: component

$\forall x$ : reservoir, $\forall n, m$ : number

$[\operatorname{value}(x$, volume, current, $n) \wedge \operatorname{value}(x$, volume, maximum, $m) \wedge$

$(n / m>0.95) \rightarrow \operatorname{state}(x$, full $)]$

This rule represents that when the current quantitative value of volume of a certain reservoir is greater than $95 \%$ of its capacity, then the state of this reservoir is full. For the case of complex components, their state is determined by conditions about the state of simpler components. This is formulated by aggregation rules such as the following example:

sort point, basin: component,

$\forall x 1$ : point, $\forall x 2$ : basin

$[$ part_of $(x 1, x 2) \wedge \operatorname{state}(x 1$, heavy-rain $) \rightarrow \operatorname{state}(x 2$, heavy-rain $)]$ 
This rule represents that if the state of a spatial point is heavy rain and it is part of a basin, then the state of the basin is also heavy rain. Note that at a certain moment, according to these rules, a complex component could have a number of different states at the same time, deduced from the states of subcomponents that have different states. In order to cope with this, we process these rules following a particular control mechanism based on relevance as it is described in the next section.

The interpretation model also is used to formulate how the value of a quantity $x$ is computed based on the values of other quantities $y_{1}, y_{2}, \ldots, y_{n}$ (when $x$ is not directly measured by sensors). We also use rules to represent this type of knowledge.

\subsection{The relevance model}

The relevance model represents knowledge to determine when a certain event is relevant to be reported to the operator. In general, we define a relevant event that produces (now or in the near future) a significant deviation of the desired state established by the goals of the management strategy for the dynamic system. The implication of this definition is that, in order to evaluate the relevance of facts, it would be necessary to predict the behavior of the dynamic system by simulation. However, based on our assumption for system modeling, we follow here a simplified and more efficient approach with approximated heuristic knowledge.

We use the predicate normal_state $(x$ : component, $y$ : state) to establish that the qualitative state of a type of component does not produce significant deviations of the desired states established by the management goals. For example, in hydrology, one of the management goals is that the water flow in river channels should not exceed the maximum capacity of channels in order to avoid floods. Thus, light rain is not relevant because, normally, it does not produce this problem. This can be represented as normal_state(point, light-rain). However, if we want to establish exceptions for this situation, we can write rules such as the following:

sortpoint : component, $\forall x:$ point, $\forall y$ : weather-forecast-region

$[$ part_of $(x, y) \wedge \neg \operatorname{state}(y$, hea $v y$-storm $) \rightarrow$ normal_state $(x$, light-rain $)]$

This rule represents that the state light rain is not relevant at a certain point, except when the weather forecast predicts heavy storm in the region of such point (because it may be the beginning of the storm).

Our notion of relevance gives also criteria to establish relevance order among events. To represent relevance order, we use the predicate more_relevant $(x, y)$ where $x$ and $y$ are states. ${ }^{2}$ For example more_relevant(state(a, heavy-rain), state(b, light-rain)) represents that the state heavy rain is more relevant that the state light rain. We use rules with this predicate in the consequent to consider particular situations. For example, the state heavy-rain at certain location $x$ can be more relevant than the same state at location $y$ because, for example, the capacity of the river channels nearby $x$ is smaller or because there are populated areas nearby $x$.

The relevance model plays the role of control knowledge in the complete model. The aggregation rules of the interpretation model are used to determine the state of complex components based on the state of single components. However, these rules need to be applied according to a certain control mechanism be-

\footnotetext{
${ }^{2}$ In order to use a formula of first-order logic using the predicate more_relevant $(x, y)$, then state $(x, y)$ must be a term and not a predicate. We use here the technique of reification McCarthy (1979), which in general consists of making a formula of firstorder language $L 1$ into a term of another first-order language $L 2$. We make atoms of $L 1$ into terms of $L 2$. We treat the predicate symbol of $\operatorname{state}(x, y)$ in $L 1$ as a function symbol in $L 2$ (whose sort is, for example, state_predicate). Then in $L 2, \operatorname{state}(x, y)$ is a term.
}

cause the same component can have different states. The relevance priority is used here for this purpose taking into account that rules that interpret qualitative states with higher priority are applied first.

\subsection{The validation model}

In real life, the measures recorded by sensors that monitor the behavior of dynamic systems present sometimes errors due to occasional malfunctions of devices or communications. For this purpose, it is important to have a model that represents knowledge able to detect typical situations that correspond to these faults. We use the predicate error( $x$ : sensor, $y$ : malfunction) to represent that a particular sensor is out of order (here, the sort malfunction represents a prefixed malfunction). The validation model contains validation rules with this predicate in the consequent. The antecedent of such rules includes conditions that analyze the values of the quantity recorded by the sensor.

This process can detect errors corresponding to certain properties of the quantity (maximum value, minimum values, maximum change in a time interval, etc.). A typical fault in the field of hydrology is a pluviometer that records a constant value for a long period. According to the behavior of the rain, it is expected a certain random behavior of the values. We can easily detect this error with a rule with this condition in the antecedent. In addition, validation rules can include conditions that analyze the coherence of sets of nearby sensors based on redundancy of measures and/or causal influences.

\subsection{Abstraction inferences}

The abstraction model also includes a prefixed set of goals that correspond to specific abstraction inferences. Each inference goal is identified by a predicate. For example, the predicate $\operatorname{abstract}(x)$ represents an inference goal that generates $x$ (an ordered list of qualitative states) by abstracting the quantitative sensor measurements. Other examples of predicates are the following: details $(x, y)$ represents an inference goal that generates a list of states $y$ that are the details of the state $x, \operatorname{causes}(x, y)$ generates the list of states $y$ that are cause of the state $x$, and effects $(x, y)$ generates the list of states $y$ that are effects of the state $x$.

We describe below the algorithms for the abstraction goals corresponding to the predicates $\operatorname{abstract}(x)$ and details $(x, y)$. These predicates are representative examples of abstraction goals (the other abstraction goals are implemented with small variations of these main algorithms or they correspond to simple inferences). The algorithm for the predicate $\operatorname{abstract}(x)$ performs a linear sequence of the following steps:

(1) Interpret measurements. Each sensor measurement is interpreted to generate quantity values of single components. This process filters measurements that correspond to sensors that present malfunctions. Here, we use the predicates observe $(x, y, z)$ from the system model and the rules with the predicate $\operatorname{error}(x, y)$ in the consequent from the validation model.

(2) Interpret quantities. For every single component its qualitative state and its trend is computed. We use the qualitative interpretation rules with the predicate $\operatorname{state}(x, y)$ in the consequent from the interpretation model. Let $R 1=\left\{S_{1}, S_{2}, \ldots, S_{n}\right\}$ be the result of this interpretation where each element $S_{i}$ is a pair $\left\langle\operatorname{state}\left(c_{i}, a_{i}\right)\right.$, trend $\left.\left(c_{i}, t_{i}\right)\right\rangle$ where $c_{i}$ is a component, $a_{i}$ is a state and $t_{i}$ is a trend.

(3) Select relevant states. Relevant states from $R 1$ are selected. For this purpose, we use rules with the predicate normal_state $(x, y)$ in the consequent from the relevance model. Let $R 2$ be the set of relevant states. 
(4) Sort states. The states of $R 2$ are sorted according to the priority for relevance. For this purpose, we use rules with the predicate more_relevant $(x, y)$ in the consequent from the relevance model. Let $R 3$ be the resulting ordered set. More relevant states are located first in $R 3$.

(5) Filter states. States corresponding to the same phenomenon are removed. We assume that this happens when a state is cause of another state. For each state $S_{i}$ in $R 3$ (following the priority order in $R 3$ ) a second state $S_{k}$ (less relevant than $S_{i}$ because $S_{k}$ is located after $S_{i}$ in $R 3$ ) is removed if $S_{k}$ is cause or effect of $S_{i}$ (through the causal relation established the predicate $\operatorname{cause}(x, y, z, u, v)$ from the system model). Let $R 4$ be the resulting filtered set.

(6) Condense states. We condense states from $R 4$ to generate a new set with a small number of more abstract states. As control criteria, we condense first the states according to the order in $R 4$ with guarantee that the most relevant states are condensed first. In this process, each time a condensed state is generated, we also generate a link that relates the condensed state and the states of single components that it abstracts. Two strategies are used for condensation:

(a) State generalization. We generate a new state of a component that generalizes a set of states of the same component. For example, the states heavy_rain and moderate_rain are generalized by the state rain. For this purpose, we use the predicate state_category $(x, y)$ from the system model. We look for the most specific category that generalizes all the states of components of the same class.

(b) Component aggregation. The goal is to condense states of single components $c_{1}, c_{2}, \ldots, c_{n}$ of the same class to generate the state of a complex component $c$. The complex component $c$ is the smallest component that includes all the single components $c_{1}, c_{2}, \ldots, c_{n}$ through the relation established by the part_of $(x, y)$ predicate from the system model. We determine the state of the component $c$ with the rules from the interpretation model that include the predicate state $(x, y)$ in the consequent. In addition, the trend and the quantification are evaluated. The quantification is calculated by comparing the size of the set of states to condense and the size of the set that includes all single components that are part of the component $c$. As a result, this condensation produces a tuple with the following format $\langle\operatorname{state}(c, s)$, trend $(c, t)$, quan$\operatorname{tification}(c, q)\rangle$.

The output of this abstraction process is an ordered set with the following format $R=\left\{\left\langle\operatorname{state}\left(c_{1}, s_{1}\right)\right.\right.$, $\operatorname{trend}\left(c_{1}, t_{1}\right), \quad$ quantifica$\left.\operatorname{tion}\left(c_{1}, q_{1}\right)\right\rangle, \quad\left\langle\operatorname{state}\left(c_{2}, s_{2}\right), \quad \operatorname{trend}\left(c_{2}, t_{2}\right), \quad\right.$ quantification $\left.\left(c_{2}, q_{2}\right)\right\rangle, \ldots$, $\left\langle\operatorname{state}\left(c_{n}, s_{n}\right)\right.$, trend $\left(c_{n}, t_{n}\right)$, quantification $\left.\left.\left(c_{n}, q_{n}\right)\right\rangle\right\}$. The elements of the set $R$ are ordered by relevance. In addition, as mentioned, each state of a complex component is linked with the set of single components that it abstracts. These links make possible to perform an inverse inference, represented by the predicate $\operatorname{details}(x, y)$. Thus, when this predicate is invoked with a value for $x$ (the state of a component), it looks for the corresponding link and returns the value $y$ with the states of single components.

\section{The presentation model}

The presentation model is used to automatically construct presentation plans, i.e. the final output description to be presented to the end-user. The presentation model represents knowledge about strategies that express how to present abstracted information. We use a knowledge-based hierarchical planner that constructs the presentation plan through a search directed by hypotheses of partial strategies that are progressively refined until the final presentation is generated. The knowledge base of the planner contains a set of operators that represent atomic presentation operations.

Fig. 2 shows a set of operators. In this example, operators generate text using templates (it is also possible to have graphic operators, as it is explained below). Each operator includes a set of conditions. In this example, the conditions establish that the operators are applicable for a particular type of component (a river section) besides other conditions about the values of quantities. In addition, the operator defines its effect with one or several presentation actions that generate part of the presentation. We consider actions to generate text and actions to generate illustrations (images, animations, graphics, etc.). In this example, the actions generate text using the action add-text( $x$ ) that incrementally constructs the text summary.

Each specific operator is associated to a general communicative goal. For example, the goal of the last operator is contrast_to_aver$\operatorname{age}(x, q)$, i.e., to show an average value that contrast to a previous reported value. In general, there are several candidate operators for a given communicative goal. Each communicative goal is defined according to certain rhetorical relations that establish the structure of discourse (Mann \& Thompson, 1988). We have identified a set of rhetorical relations applicable in our context of dynamic system surveillance that include relations such as: elaboration, contrast, exemplify, list, cause, preparation, etc. Table 3 shows a list of communicative goals that implement such relations. The table includes examples of text sentences for these communicative goals.

To generate a presentation, the planner searches for applicable operators. We use a hierarchy of discourse patterns to direct this search. The hierarchy makes more efficient the search process and helps to generate coherent presentations. Our presentation planner uses a simplified version of Hierarchical Task Network (HTN) planning (Ghallab, Nau, \& Traverso, 2004). Besides operators, an HTN planner includes planning-tasks and planning-methods. In our approach, each planning-task corresponds to a communicative goal (what to present) and each planning-method corresponds to a discourse pattern (how to present it). In our model, a discourse pattern is a presentation template that helps to keep the coherence of the presentation. Each pattern defines a partial presentation strategy at certain level of abstraction.

Fig. 3 shows examples of discourse patterns. The first extract illustrates a discourse pattern for the communicative goal inform $(x)$. In general, several different patterns can be associated to the same goal. The pattern defines four sub-goals: summarize_state $(x)$, elaborate_state $(x)$, elaborate_causes $(c)$ and elaborate_effects( $e$ ). The pattern includes also conditions similar to the conditions for operators whose purpose is to establish when the pattern is applicable. Conditions can include logical expressions

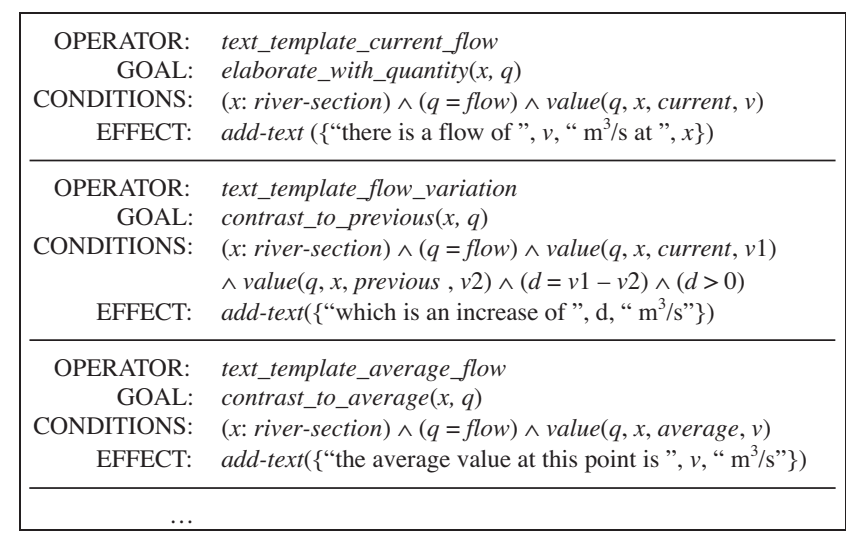

Fig. 2. Examples of planning-operators in the presentation model. 
Table 3

Examples of communicative goals associated to text templates.

\begin{tabular}{|c|c|}
\hline Communicative goal & Example of generated sentence (translated into English) \\
\hline summarize_state $(x)$ & High flow in some parts of the river Ebro \\
\hline elaborate_with_quantity $(x, q)$ & The flow of river Ebro at Tortosa is $1285 \mathrm{~m}^{3} / \mathrm{s}$ \\
\hline elaborate_with_count $(x)$ & High flow in three rivers of the Ebro Basin \\
\hline elaborate_with_enumerate $(x)$ & High flow in the river Ebro, the river Jucar and the river Segura \\
\hline list_maximum $(x, q)$ & The river Ebro has the maximum flow \\
\hline list_next_maximum $(x, q)$ & The river Arga is the next river with maximum flow \\
\hline contrast_to_average $(x, q)$ & The average flow at this point of the river is $309 \mathrm{~m}^{3} / \mathrm{s}$ \\
\hline contrast_to_previous $(x, q)$ & Compared to the previous value, this means an increase of $130 \mathrm{~m}^{3} / \mathrm{s}$ \\
\hline contrast_to_maximum $(x, q)$ & This value corresponds to the $78 \%$ of its capacity \\
\hline exemplify_with_quantity $(x)$ & For example, the flow of river Ebro at Tortosa is $1285 \mathrm{~m}^{3} / \mathrm{s}$ \\
\hline exemplify_with_next_quantity $(x)$ & Also, the flow of river Ebro at Asco is $973 \mathrm{~m}^{3} / \mathrm{s}$ \\
\hline prepare_causes & The following behavior upstream can be highlighted \\
\hline prepare_effects & The following behavior downstream can be highlighted \\
\hline summarize_behavior $(x)$ & Light rain in the Ebro basin in the last $24 \mathrm{~h}$ \\
\hline elaborate_with_quantity_behavior $(x, q)$ & In the last $24 \mathrm{~h}$, the accumulated rain at Algemesí is $27 \mathrm{~mm}$ \\
\hline list_maximum_behavior $(x, q)$ & In the last $24 \mathrm{~h}$, the place with maximum accumulated rain is Algemesi \\
\hline list_next_maximum_behavior $(x, q)$ & The place with next maximum accumulated rain is Albalat \\
\hline
\end{tabular}

with predicates related to the structure and behavior of the dynamic system (for example, value $(x, y, z, t)$, part_of $(x, y)$, etc.). In the examples, value $(q, x$, current, $v$ ) is used to identify the current value of a quantity, single_component $(y)$ indicates that $y$ must be a single component and quantity $(y, q)$ represents that $q$ is a quantity of the component $y$. In addition, conditions also could include predicates related to previous decisions about presentation elements (such as the decision about the layout). Conditions can also include predicates that invoke abstraction goals. These predicates gather additional information about the dynamic system (for example, the predicates causes $(x, c)$ and effects $(y, e)$ ). Abstraction goals are invoked backwards when the presentation planner checks the conditions of a candidate pattern.

Using a presentation model as a knowledge base with operators and patterns is fairly flexible. It permits the discourse planner to consider different types of presentation strategy, and it can be easily extended to include new strategies. As the examples illustrate, the discourse patterns are domain independent so that they can be reused (wholly or partially) for different dynamic systems. In contrast, operators are domain dependent. For example, they define specific text templates using specific words and sentences that are domain specific and need to be defined with the help of domain experts. In order to have more general operators, natural language generation (NLG) techniques could be used instead of templates (as it is done for example in Molina \& Stent, 2010) and further research could help to identify common presentation strategies at operator level that could be generalized for different dynamic systems.

\begin{tabular}{|c|c|}
\hline $\begin{array}{r}\text { PATTERN: } \\
\text { GOAL: } \\
\text { CONDITIONS: } \\
\text { DISCOURSE: }\end{array}$ & $\begin{array}{l}\text { describe_with_causes_and_effects } \\
\text { inform }(x) \\
\text { causes }(x, c) \text { reffects }(y, e) \\
\{\text { summarize_state }(x), \text { elaborate_state }(x), \\
\text { elaborate_causes }(c), \text { elaborate_effects }(e)\}\end{array}$ \\
\hline $\begin{array}{r}\text { PATTERN: } \\
\text { GOAL: } \\
\text { CONDITIONS: } \\
\text { DISCOURSE: }\end{array}$ & $\begin{array}{l}\text { describe_state_of_single_component } \\
\text { elaborate_state }(x) \\
(\text { state }(y, s) \in x) \wedge \text { single_component }(y) \wedge q u a n t i t y(y, q) \\
\{\text { elaborate_with_quantity }(y, q), \\
\text { contrast_to_previous }(y, q), \text { contrast_to_average }(y, q)\}\end{array}$ \\
\hline $\begin{array}{r}\text { PATTERN: } \\
\text { GOAL: } \\
\text { CONDITIONS: } \\
\text { DISCOURSE: }\end{array}$ & $\begin{array}{l}\text { describe_state_of_complex_component } \\
\text { elaborate_state }(x) \\
(\text { state }(y, s) \in x) \wedge \neg \text { single_component }(y) \\
\{\text { elaborate_with_count }(x), \text { exemplify_with_quantity }(x)\}\end{array}$ \\
\hline
\end{tabular}

Fig. 3. Examples of discourse patterns in the presentation model.
To construct a presentation plan our planner searches the space of possible plans using the discourse patterns (Fig. 4 and Table 4). During the search, the presentation planner repeatedly (a) selects a candidate discourse pattern for the current goal, (b) checks if the conditions of the candidate discourse pattern are satisfied and sometimes, as a consequence of this, gathers additional abstracted information, and (c) refines the communicative goal with subgoals. This process continues for each communicative goal until it can be carried out by an operator that satisfies its conditions. Each operator produces a certain part of the presentation. The whole presentation is formed as the sequence of all design decisions done by the operators.

As mentioned, operators can include other type of actions to generate multimedia presentations. For example, it is possible to generate 3D animations in a virtual terrain by using specific presentation primitives that a viewer for virtual terrains can interpret.

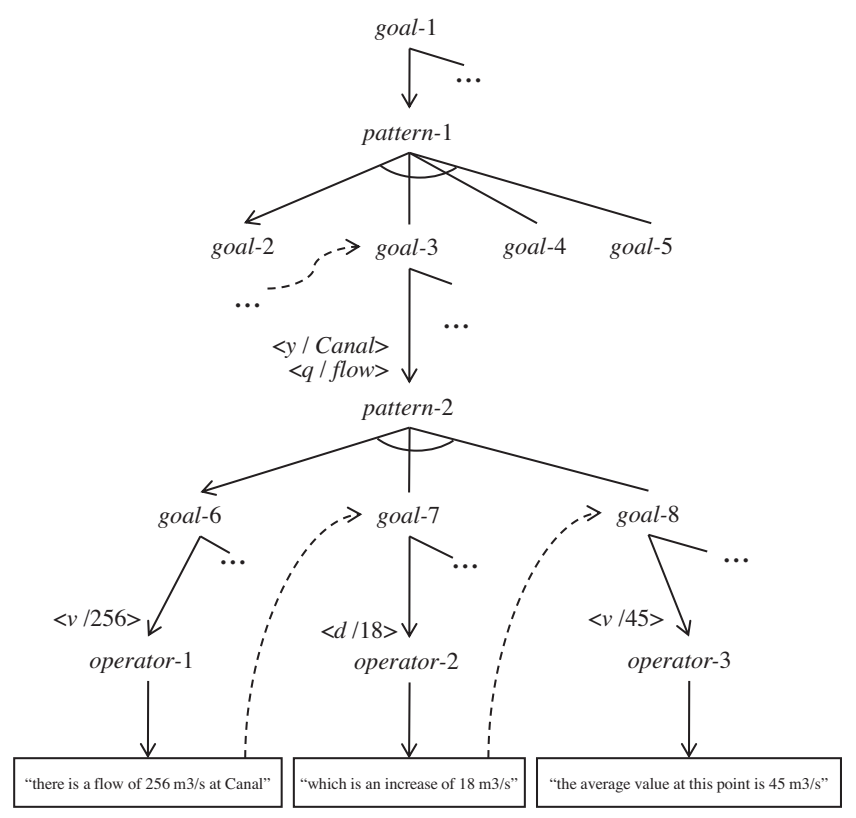

Fig. 4. Example of partial search tree developed during the presentation planning process. The tree includes several nodes whose content is shown in Table 3. This example uses the operators and patterns shown in Figs. 2 and 3 respectively. Some of the variable substitutions are shown in the figure (e.g., $\langle d / 18\rangle$ means that variable $d$ is substituted by the value 18 , as it is done, for example, by unification in automated theorem proving). 
Table 4

Content of nodes corresponding to the tree shown in Fig. 3.

\begin{tabular}{ll}
\hline Node & Content \\
\hline goal-1 & inform( $(\langle$ state $($ Canal,high-flow),trend(Canal,increase $)\rangle)$ \\
goal-2 & summarize_state $(\langle$ state $($ Canal,high-flow),trend(Canal,increase $)\rangle)$ \\
goal-3 & elaborate_state $(\langle$ state $($ Canal,high-flow $), t r e n d(C a n a l$, increase $)\rangle)$ \\
goal-4 & elaborate_causes $(\langle$ state $($ Canal,high-flow),trend(Canal,increase $)\rangle, \ldots)$ \\
goal-5 & elaborate_effects $(\langle$ state $($ Canal,high-flow),trend(Canal,increase $)\rangle, \ldots)$ \\
goal-6 & elaborate_with_quantity $($ Canal, flow $)$ \\
goal-7 & contrast_to_previous $($ Canal, flow) \\
goal-8 & contrast_to_average $($ Canal, flow $)$ \\
pattern-1 & describe_with_causes_and_effects \\
pattern-2 & describe_state_of_single_component \\
operator-1 & text_current_flow \\
operator-2 & text_flow_variation \\
operator-3 & text_average_flow \\
\hline
\end{tabular}

Primitives locate certain elements in a 3D map (points, areas, point sets) and, also, text can be presented in information globes associated to certain locations. Examples of these primitives include locate_point $(x)$, locate_area $(x)$, locate_point_set $(x)$, show_distance $(x, y)$, show_section $(x, y)$, show_text_globe $(x, y)$, show_orography $(x)$, etc. The viewer uses a cartographic database with raster and vector data corresponding to digital elevation models about terrains, orto-photographies, etc. An example of planning operator with this type of presentation primitives is the following:

OPERATOR: current_flow_on_virtual_terrain

GOAL: elaborate_with_quantity $(x, q)$

CONDITIONS: $\quad(x$ : river-section $) \wedge(q=$ flow $) \wedge \operatorname{value}(q, x$, current, $v$ )

EFFECT: \{locate_point $(x)$, show_text_globe $(x,\{$ "there is a flow of ", $v$, " $\mathrm{m}^{3} / \mathrm{s}$ at ", $\left.\left.\left.x\right\}\right)\right\}$

\section{Evaluation}

In order to evaluate our method, we used a real world domain with enough complexity and available data to experiment with different models. We used the domain of hydrology to evaluate the validity of our method and its practical utility. This domain is especially appropriate because (1) there are available large sets of measures (historical databases and in real time) about the rivers' behavior in Spain thanks to a national information system called SAIH (SAIH: Spanish acronym for Automatic Information System in Hydrology), and (2) this information is used to make decisions according to different goals such as flood alerts, water management, hydroelectric energy, sensor validation, etc.

The SAIH system includes a sensor network to record hydrologic measures such as rainfall at certain geographical points, flows and/ or water level of river channels, water levels of reservoirs, etc. The SAIH system consists of nine control centers in Spain, one for each main river basin (Ebro, Tajo, Júcar, Segura, etc.). Control centers receive in real time hydrologic data recorded by sensors. Control centers process and store this information in databases. The Ministry of Environment of Spain coordinates and integrates part of the recorded data in a global database. Besides other information, the database includes time series with values of the last $T$ hours ( $T=24 \mathrm{~h}$ and $\Delta T=1 \mathrm{~h}$ or $30 \mathrm{~min}$ ).

We developed three different models for this domain according to three different management goals: flood risk, water management, and sensor validation. For example, the flood risk management goal is to avoid river floods. In this case, control actions are oriented to operate reservoirs to avoid problems produced by floods and, when problems cannot be avoided, to send information to public institutions in order to plan defensive actions. For this management goal, the behavior summaries report relevant information of the river basin from the point of view of potential or existing floods such as the presence of significant rain or high flow at certain locations. We constructed two versions for the flood risk model. The first version includes a presentation model combining text (in the form of headlines and text globes) and 3D animations on a virtual terrain. The second version includes a presentation model combining text with interactive maps and graphics with time series.

We formulated a common model for the dynamic system shared by the rest of the models. This includes different types of sensors (e.g., pluviometers, flow-sensors, level-sensors) and components (e.g., river section, reservoir, rain point, river, basin, region and nation). These components are related with structural relations (e.g., part-of). In addition, causal relations describe the water behavior from rain components to downstream components. The resulting model includes 14,337 elements distributed in the following way: 1864 values of sort sensor, 2230 values of sort component, 2229 instances of predicate part_of $(x, y), 1864$ instances of predicate measure $(x, y, z), 2068$ instances of $\operatorname{value}(x, y, t, v)$ (e.g., maximum value and average value), 2295 instances of cause $(x, y, z, u, t)$ for pluviometers, 687 instances of cause $(x, y, z, u, t)$ for river channels.

To develop such a model, we applied a semi-automatic knowledge acquisition process supported by software tools (developed in our own research group) using different information sources. Examples of these information sources include (1) geographic information such as raster files with digital elevation models and vector data files with rivers, reservoirs, basins, dams, administrative limits (provinces, regions, etc.), and (2) web applications with publicly available information, such as web pages with hydrologic information provided by local SAIH control centers, and the web site http:// www.geonames.org that provides names for geographical locations. In this process, we performed certain operations (spatial analysis, statistical analysis and text processing) to capture and represent knowledge from the different information sources.

We developed three abstraction models and three presentation models corresponding to the three management goals (flood risk, water management and sensor validation). The domain abstraction models include in total 206 logic clauses. The domain presentation models include 294 logic clauses. The most complex presentation model is the model for the case of flood risk. The first version of the presentation model includes 38 operators and 42 patterns, which generates 3D animations on a virtual terrain (Fig. 5). The second version of the presentation model for text and, 2D maps and graphics includes 103 operators and 142 patterns.

We evaluated the models with data corresponding to measures of SAIH sensors. This evaluation was done in two phases. In the first phase, we prepared a set of representative cases with a simulator that covered different hydrologic episodes of $24 \mathrm{~h}$. The method was applied every hour to generate the corresponding summaries. For example, one of these episodes included data for 57 pluviometers, 117 flow sensors and 71 volume sensors (since we have 24 measures for each sensor, this includes a total number of 5880 measures). A representative sample of the generated summaries was evaluated with the help of a human expert in hydrology. For this evaluation, we used a method post-edition method (as it is done for example in Turner, Sripada, \& Reiter, 2009). For each generated summary, we asked the expert to write a new text summary describing the hydrologic situation by reusing our generated summary. Then, we compared the generated summary and the summary written by the expert. We computed the percentage of generated text for each generated summary that was present in the summary written by the expert. The result of this evaluation generated an average value of $83 \%$ which was considered satisfactory. Some of the differences between summaries were related to verb tenses and syntax rules about specific names of locations. 


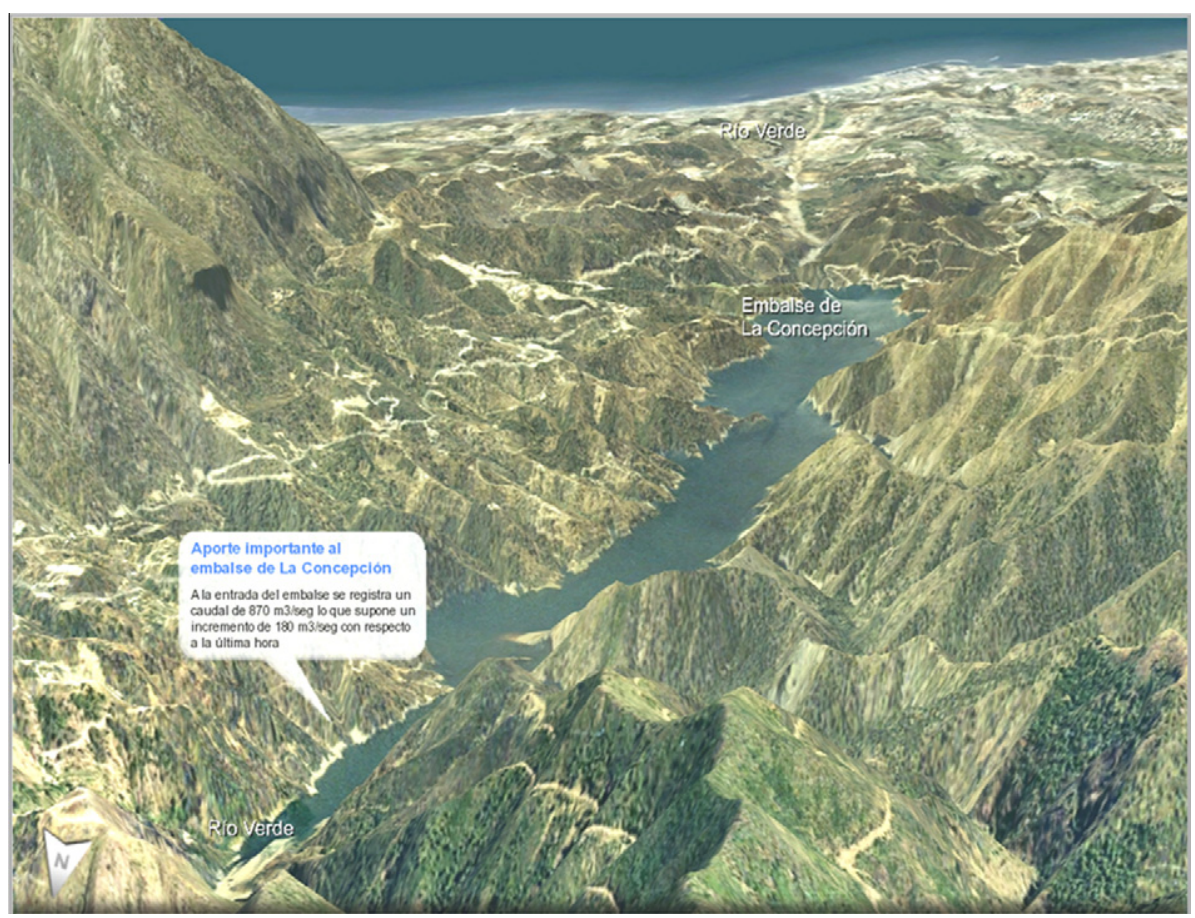

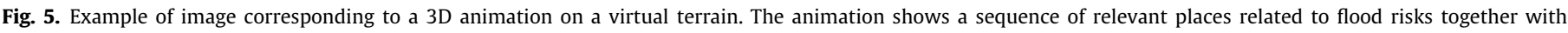
explanatory text globes with details.

For the second phase, we validated the models by developing a web application, called VSAIH (Fig. 6), that operated on line with sensor data provided by the SAIH system. The application includes the three models (flood risks, water management and sensor validation) and generates summaries by processing every hour 44,736 numerical measures. This corresponds to 1864 sensors and, for each sensor, a time series for the last $24 \mathrm{~h}$ (one value per hour). The method generates summaries for each hour in less than $30 \mathrm{~s}$. The method was implemented in Prolog language, both inference procedures and knowledge bases. We validated the correctness of the models by continuous operation of this application for more than 1 year with the help of three experts in hydrology who refined details of the summaries.

We estimated the practical utility of this application by comparing the VSAIH application with existing web applications about hydrologic information. For this purpose, we selected 10 representative summaries provided by VSAIH and measured the effort (in time) to get the reported information by other web sources. We mainly used three different types of web sources: (1) the web site of the Ministry of Environment of Spain with information about the measures recorded by sensors, (2) the web site of the National Agency of Meteorology of Spain, and (3) the web site of SAIH control centers about river basins, which includes additional and more detailed information about sensors (historical values, detailed maps, etc.). The result of this evaluation produced a maximum value for the estimated effort of about $5 \mathrm{~h}$ (exactly, $4 \mathrm{~h}$ and $46 \mathrm{~min}$ ). This represents the time that operators could save by using the VSAIH application, which is a significant value, especially in the presence of hydrologic emergencies.

\section{Related work}

Our method integrates various types of artificial intelligence techniques related to different fields: system modeling, explanation systems, summarization, and generation of multimedia presentations. In the following, we compare our method with related work in each one of these fields. We designed our representation for the system model to capture qualitative descriptions as it is done in physical reasoning. In general, physical reasoning uses qualitative descriptions that contrast to scientific computing that usually aims at achieving a high degree of numerical accuracy (Davis, 2008). The qualitative approach is used in qualitative simulation (de Kleer \& Brown, 1984; Forbus, 1984; Kuipers, 2001), representation languages such as CML (Bobrow et al., 1996) and DME (Iwasaki \& Low, 1993) and ontologies for physical systems (e.g., (Borst et al., 1995; Gruber \& Olsen, 1994). As a main difference with these approaches, we designed our representation to simulate abstraction reasoning (by qualitative interpretation, aggregation and generalization) instead of other tasks that usually require more precision (e.g., diagnosis or prediction). Thus, compared to other representation for physical reasoning our representation is simpler and, therefore, more efficient for both inference and knowledge acquisition.

Our method is also related to automatic tools that generate explanations about the behavior of dynamic systems. As mentioned, the method of Gautier and Gruber is a representative example of this type of tool (Gruber \& Gautier, 1993). This tool uses a system model represented in DME language (Iwasaki \& Low, 1993) to generate text explanations in a dialog with the user that help to understand the behavior a space shuttle's reaction control system. The main difference with our method is that this tool generates explanations of single events, with limited capabilities for abstraction. Instead, our method includes a model-based abstraction component, which is useful to generate descriptions that abstract large amounts of related events. The method of Gautier and Gruber defines relevance based on qualitative state transitions. Our method also uses qualitative states to distinguish relevant situations. However, our method includes an additional definition for relevance. Our method uses a notion of relevance based on the distance between the system state and the management goals. This provides a clear criterion to establish degrees of relevance. Also, it is used as control criteria to use abstraction knowledge for summarization. 


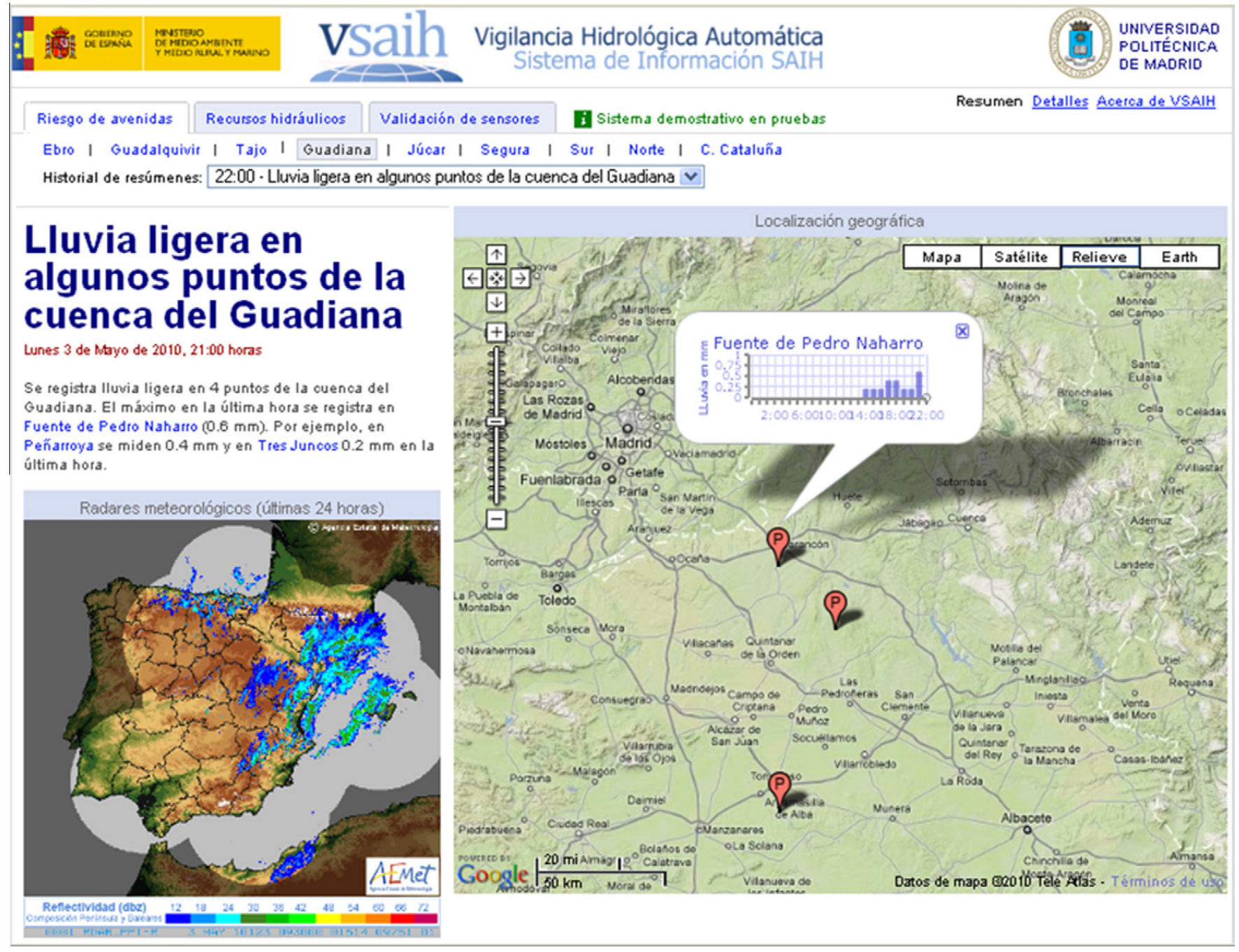

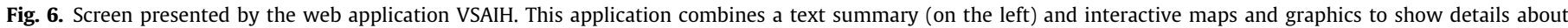

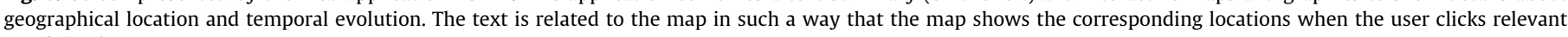
words in the text.

Our method is also related to data-to-text systems that use techniques for summarizing time series data. For example, our work presents commonalities with the SumTime project in the domain of weather forecast (Reiter et al., 2005). The SumTime approach has been applied to the domain of medicine (Hunter, Gatt, Portet, Reiter, \& Sripada, 2008) and gas turbines (Yu, Reiter, Hunter, \& Mellish, 2007). The FOG system (Goldberg, Driedger, \& Kittredge, 1994) is a previous data-to-text system that converts weather data into forecast text.

Our method and SumTime generate descriptions that summarize time series. The main difference is that SumTime is restricted to text generation, while our system can generate also descriptions with 3D animations and other multimedia presentations. SumTime includes a natural language generation module for text generation. Instead, our method uses a hierarchical planner with operators that follow a template-based approach for text generation (Reiter, 1995). Our planner can combine efficiently text with other media which is important for complex and large systems with thousands of time series.

In the field of summarization, abstraction techniques go from domain dependent approaches to domain independent solutions based on more general approaches (e.g., clustering or statistic analysis) (Maybury, 1993). For example, SumTime follows a domain dependent approach that abstracts time series based on a general pattern matching method (Knowledge-based Temporal Abstraction) (Shahar, 1997). SumGen is another case of data-to-text system (Maybury, 1995). This approach receives as input data a set of events (e.g., events produced by a battle simulator) and produces text descriptions that summarize the events. SumGen uses a general representation of events and a domain model to abstract events. As SumTime and SumGen, our approach for abstraction is also domain dependent but it uses a more specific representation for dynamic systems (e.g., components, states, quantities, etc.). Therefore, it can be easier reused for different dynamic systems, and it can be more efficient than general approaches. In contrast, the applicability of our method is limited to a certain type of dynamic systems that can be described with a component approach.

Our method is also related to multimedia presentation systems. For example, our method shares some general ideas about presentation planning and rhetorical relations as it is done by WIP (André \& Rist, 1993; Wahlster, André, Finkler, Profitlich, \& Rist, 1993) and COMET (McKeown \& Feiner, 1990). The system of Kerpedjiev in the field of meteorology (Kerpedjiev, 1992) follows a similar planning approach. The goal of COMET and WIP is to generate text explanations accompanied with static illustrations that help users to manipulate a device (e.g., a radio receiver-transmitter or a coffee machine). We designed our method for a different task: generating text explanations and interactive illustrations that help users to 
understand the behavior of a complex dynamic system (e.g., a network of river basins). Compared to the planners used by these systems, our presentation planner follows a different approach. WIP constructs a presentation based on an explicit representation of the presenter's intentions and the effects on the receiver. In contrast, our approach is closer to design planners used for configuration tasks (Agosta, 1995; Brown \& Chandrasekaran, 1989). Our planner constructs a presentation based on partial design decisions in a search directed by partial discourse strategies. Our experience in the domain of hydrology shows that our solution is efficient to construct automatically presentations for dynamic systems. In addition, it is flexible enough to easily change parts of the strategies for presentation according to new requirements.

A different approach for presentation generation is followed by ILEX (O’Donnell, 2000). ILEX generates presentations about pieces of a museum with text summaries and images. ILEX uses a semantic network to represent knowledge about pieces of art and constructs a presentation using a particular notion of relevance. ILEX uses degrees of relevance based on the distance between facts through relations (such as generalization, contrast, etc.). Our method and ILEX generate presentations that combine text and illustrations. However, they are designed for different tasks with different knowledge representation and summarization procedures (for example, ILEX does not summarize behavior).

\section{Conclusions}

In this article, we have described a method for generating descriptions that summarize the behavior of a dynamic system. We have designed this method for a type of dynamic system that is monitored with the help of sensors to help human operators to make decisions according to certain management goals. The generated descriptions include both text and graphics such as maps, 3D animations on virtual terrains and 2D graphics for temporal series. Our method integrates various types of artificial intelligence techniques related to different fields: system modeling, explanation systems, summarization, and generation of multimedia presentations. The main original contributions of the method are the following:

- To our knowledge, our method is the first method for the task of generating descriptions with coordinated text and graphics that summarize thousands of quantitative measures of the behavior of a dynamic system. Our method has been validated in a real world domain. Other related methods correspond to different tasks or partial solutions (e.g., they generate only text, they abstract data from few sensors or they are experimental prototypes).

- We designed a general representation for dynamic systems (e.g., sensors, components, quantities, states, etc.) to support abstraction and presentation planning. This provides generality of the method (it is applicable to different dynamic systems) and it also provide reusable declarative expressions that make it easier for a developer to build the domain models for a new application. For example, the representation provides reusable predicates and axioms to describe the dynamic system and general rules and planning operators for abstraction and presentation. In contrast, the applicability of our method is limited to a kind of dynamic system that can be modeled with our representation.

- We also designed specific algorithms for inference. For example, we designed original procedures to generate efficiently abstractions by using the representation of the dynamic system. Here, we defined a particular notion of relevance (based on the distance between the system state and the management goals) that plays a role of control knowledge in the global abstraction inference. In addition, we used and adapted existing planning algorithms (e.g., planning algorithms for design tasks and HTN planning) to define an efficient solution for the generation of multimedia presentations.

- The application that we developed in the hydrologic domain showed that our method was able to generate efficiently useful summaries by using approximate knowledge that was possible to acquire with acceptable effort. We especially confirmed this by building several real-world models using available information sources and semi-automatic procedures. Our representation was also flexible enough to easily change parts of the strategies for presentation according to changes in the requirements.

One of the practical results of this work is the web application VSAIH that we developed for the Ministry of Environment in Spain (Dirección General del Agua, Ministerio de Medio Ambiente, Medio Rural y Marino). This application uses the method described in this article to generate in real time summaries of data from thousands of hydrologic sensors. Part of our future work includes to extend our method (for example, by including flexible dialog mechanisms that improve the user-system interaction) and to apply our representation for other types of dynamic systems. For example, we plan to use this solution to summarize data related to aircrafts and radars in the context of battle scenarios (simulated or real) during military missions.

\section{Acknowledgments}

This work was possible thanks to the support of the Ministry of Environment of Spain (Dirección General del Agua, Ministerio de Medio Ambiente, Medio Rural y Marino). The work was also partially supported by the Ministry of Science and Innovation of Spain within the VIOMATICA project (TIN2008-05837/TIN) and the E-VIRTUAL project (REN2003-09021-C03-02). The authors thank the member of our research group Luis Garrote who helped with valuable comments the design of the method. The authors also thank the members of our research group: Javier Sánchez-Soriano for the implementation of the web application that helped to evaluate the final version of the method, and Enrique Parodi for the implementation of the 3D graphical viewer. The implementations of earlier versions of the method were made possible with the help of the members of our research group Sandra Lima, Alfonso Sánchez, Javier Bracicorto, María del Carmen Martín, Nuria Andrés and Sergio Gayoso. The authors thank Amanda Stent for her useful comments on earlier versions of this article.

\section{References}

Agosta, J. M. (1995). Formulation and implementation of an equipment configuration problem with the SIPE-2 generative planner. In Proceedings AAAI-95 spring symposium on integrated planning applications.

André, E., \& Rist, T. (1993). The design of illustrated documents as a planning task. In M. Maybury (Ed.), Intelligent Multimedia Interfaces (pp. 94-116). AAAI Press.

Bobrow, D., Falkenhainer, B., Farquhar, A., Fikes, R., Forbus, K. D., Gruber, et al. (1996). A compositional modeling language. In Proceedings 10th international workshop on qualitative reasoning about physical systems (pp. 12-21).

Borst, P., Akkermans, J. M., Pos, A., \& Top, J. L. (1995). The PhysSys ontology for physical systems. In Bredeweg, R. (Ed.), Working papers ninth international workshop on qualitative reasoning QR'95, Amsterdam, the Netherlands (pp. 1121).

Brown, D., \& Chandrasekaran, B. (1989). Design problem-solving: Knowledge structures and control strategies. Morgan Kaufman.

Davis, E. (2008). Physical reasoning. In F. van Harmelen, V. Lifschitz, \& B. Porter (Eds.), Handbook of knowledge representation (pp. 597-620). Oxford: Elsevier.

Forbus, K. D. (1984). Qualitative process theory. Artificial Intelligence, 24, 85-168.

Ghallab, M., Nau, D., \& Traverso, P. (2004). Automated planning: Theory and practice. Morgan Kaufman. 
Goldberg, E., Driedger, N., \& Kittredge, R. I. (1994). Using natural language processing to produce weather forecast. IEEE Intelligent Systems and Their Applications, 9(2).

Gruber, T. R., \& Gautier, P. O. (1993). Machine-generated explanations of engineering models: A compositional modelling approach. In Proceedings of the 13th international joint conference on artificial intelligence, Chambery, France (pp. 1502-1508).

Gruber, T. R., \& Olsen, G. R. (1994). An ontology for engineering mathematics. In J. Doyle, P. Torasso, \& E. Sandewall (Eds.), Fourth international conference on principles of knowledge representation and reasoning. Germany: Bonn.

Hunter, J., Gatt, A., Portet, F., Reiter, E., \& Sripada, S. (2008). Using natural language generation technology to improve information flows in intensive care units. In Proceedings 5th conference on prestigious applications of intelligent systems.

Iwasaki, Y., \& Low, C. (1993). Model generation and simulation of device behavior with continuous and discrete changes. Intelligent Systems Engineering, 1(2).

Kerpedjiev, S. M. (1992). Automatic generation of multimodal weather reports from datasets. In Proceedings Third Conference on Applied Natural Language Processing, Trento, Italy (pp. 48 - 55).

de Kleer, J., \& Brown, J. (1984). A qualitative physics based on confluences. Artificial Intelligence, 24, 7-83.

Kuipers, B. (2001). Qualitative simulation. In R. A. Meyers (Ed.), Encyclopedia of physical science and technology (3rd ed.) (pp. 287-300). NY: Academic Press.

Mann, W. C., \& Thompson, S. A. (1988). Rhetorical structure theory: Toward a functional theory of text organization. Text Journal, 8(3), 243-281.

Maybury, M. T. (1993). Automated event summarization techniques. In EndresNiggemeyer, B., Hobbs, J., Sparck Jones K., (Eds.), Workshop on Summarising Text for Intelligent Communication, Dagstuhl Seminar Report (9350). Dagstuhl, Germany.

Maybury, M. T. (1995). Generating summaries from event data. Information Processing and Management: An International Journal, 31(5), 735-751.

McCarthy, J. (1979). First order theories of individual concepts and propositions. In Hayes, J. E., Michie, D., Mikulich L. I. (Eds.), Machine intelligence, vol. 9 (pp. 129148).

McKeown, K. R., \& Feiner, S. K. (1990). Interactive multimedia explanation for equipment maintenance and repair. In Proceedings DARPA speech and language workshop (pp. 42-47).
Meinke, K., \& Tucker, J. V. (1993). Many-sorted logic and its applications. Chichester, England: John Wiley \& Sons, Inc.

Molina, M., \& Flores, V. (2006a). A knowledge-based approach for automatic generation of summaries of behavior. In J. Euzenat \& J. Domingue (Eds.) Artificial intelligence: Methodology, systems, and applications. Proceedings 12th international conference AIMSA 2006, Lecture notes in artificial intelligence, Springer Verlag, LNAI 4183 (pp. 265-274). Varna, Bulgaria.

Molina, M., \& Flores, V. (2006b). Generating adaptive presentations of hydrologic behavior. In E. Corchado, H. Yin, V. Botti, \& C. Fyfe (Eds.), Intelligent data engineering and automated learning IDEAL 2006. Proceedings 7th international conference IDEAL 2006, Lecture notes in computer science, Springer Verlag, LNCS 4224 (pp. 896-903). Burgos, Spain.

Molina, M., \& Flores, V. (2008). A presentation model for multimedia summaries of behavior. In Proceedings of the 2008 international conference on intelligent user interfaces (IUI). Canary Islands, Spain.

Molina, M., \& Stent, A. (2010). A Knowledge-based method for generating summaries of spatial movement in geographic areas. International Journal on Artificial Intelligence Tools, 19(4), 393-415.

O’Donnell, M. (2000). Intermixing multiple discourse strategies for automatic tex composition. Revista Canaria de Estudios Ingleses, 40 (April). Special Issue on Intercultural and Textual Approaches to Systemic-Functional Linguistics.

Reiter, E. (1995). NLG vs. templates. In Proceedings fifth european workshop on natural language generation.

Reiter, E., Sripada, S., Hunter, J., Yu, J., \& Davy, I. (2005). Choosing words in computer-generated weather forecasts. Artificial Intelligence, 167, 137-169.

Shahar, Y. (1997). A framework for knowledge-based temporal abstraction. Artificial Intelligence, 90(1-2), 79-133.

Turner, R., Sripada, S., \& Reiter, E. (2009). Generating approximate geographic descriptions. In Proceedings 12th European workshop on natural language generation, Athens, Greece.

Wahlster, W., André, E., Finkler, W., Profitlich, H.-J., \& Rist, T. (1993). Plan-based integration of natural language and graphics generation. Artificial Intelligence 63, 387-427.

Yu, J., Reiter, E., Hunter, J., \& Mellish, C. (2007). Choosing the content of textual summaries of large time-series data sets. Natural Language Engineering, 13(1) 25-49. 